\title{
Specific complex formation between yeast RAD6 and RAD18 proteins: a potential mechanism for targeting RAD6 ubiquitin-conjugating activity to DNA damage sites
}

\author{
Véronique Bailly, ${ }^{1,3}$ John Lamb, ${ }^{1,4}$ Patrick Sung, ${ }^{2,3}$ Satya Prakash, ${ }^{2,3}$ and Louise Prakash ${ }^{1,3,5}$ \\ ${ }^{1}$ Department of Biophysics and ${ }^{2}$ Department of Biology, University of Rochester, Rochester, New York 14642 USA; ${ }^{3}$ Sealy \\ Center for Molecular Science, University of Texas Medical Branch, Galveston, Texas 77555-1061 USA
}

The RAD6 gene of Saccharomyces cerevisiae encodes a ubiquitin-conjugating enzyme that is required for postreplication repair of UV-damaged DNA, DNA damage induced mutagenesis, sporulation, and amino-end rule protein degradation. RAD6 interacts physically with the UBR1 gene product in carrying out the multiubiquitination of amino-end rule proteolytic substrates. In mediating postreplication repair, it has remained unclear whether RAD6 acts in a pleiotropic manner distal from the site of DNA damage or is targeted to the damage site via interaction with another repair component. Here, we show that RAD6 forms a specific complex with the product of the DNA repair gene RAD18. The biological significance of this interaction is attested by the observation that overproduction of the rad6 Ala-88 mutant protein, which lacks ubiquitin-conjugating activity but retains the ability to interact with RAD18 protein, confers a high level of UV sensitivity on wild-type $R A D^{+}$cells that can be corrected by the concomitant overexpression of $R A D 18$. We demonstrate that whereas RAD6 has no affinity for DNA, RAD18 binds single-stranded DNA. Thus, association of RAD6 with RAD18 could provide a means for targeting RAD6 to damage-containing DNA regions, where the RAD6 ubiquitin-conjugating function could modulate the activity of a stalled DNA replication machinery. We also show that RAD6 forms separate complexes with RAD18 and with UBR1, and the extremely conserved amino terminus of RAD6 that is required for complex formation with UBR1 is dispensable for complex formation with RAD18.

[Key Words: RAD6 gene; RAD18 gene; Saccharomyces cerevisiae; DNA repair; ubiquitin-conjugating enzyme]

Received January 5, 1994; revised version accepted February 22, 1994.

Ultraviolet (UV)-induced DNA photoproducts present a block to the DNA replication machinery. As a result, during replication of a UV-damaged genome, singlestranded gaps are left in the newly synthesized DNA strand opposite the UV lesions. The postreplication repair pathways function to fill in the damage-associated gaps, allowing complete DNA replication. In the yeast Saccharomyces cerevisiae, the postreplication repair process is controlled by the RAD6 gene (Prakash 1981). Interestingly, rad 6 mutants are not only highly sensitive to DNA-damaging agents, but they are also defective in UV-induced mutagenesis and sporulation, and they exhibit severe growth deficiencies (Prakash et al. 1993).

The RAD6 gene product is a member of the large family of ubiquitin-conjugating (E2) enzymes in S. cerevi-

${ }^{4}$ Present address: Department of Molecular Biology and Genetics, Johns Hopkins Medical School, Baltimore, Maryland 21205 USA.

${ }^{5}$ Corresponding author. siae. Ubiquitin is a 76-residue polypeptide present in all eukaryotes, and its conjugation to cellular proteins targets them for degradation by an ATP-dependent, multisubunit protease (for review, see Jentsch 1992). Prior to conjugating ubiquitin to proteolytic substrates, RAD6 forms a thioester linkage with ubiquitin, a reaction catalyzed by the ubiquitin-activating enzyme El. The transfer of ubiquitin from RAD6 onto protein substrates can occur without any other protein cofactor (Sung et al. 1988), or it may require an additional component known as the ubiquitin protein ligase E3 (Sung et al. 1991a). In the E3-dependent mode of protein degradation, substrates bearing certain "destabilizing" residues at their amino termini are bound by E3, which also physically interacts with RAD6 protein (Dohmen et al. 1991; Watkins et al. 1993) and thus enables RAD6 to catalyze multiubiquitination of the bound substrate (Sung et al. 1991a; Watkins et al. 1993).

To evaluate the biological significance of the RAD6 
ubiquitin-conjugating activity, we altered cysteine- 88 residue in RAD6, the site of thioester formation with ubiquitin, by site-directed mutagenesis to different amino acids (Sung et al. 1990, 1991b). Mutant rad6 proteins containing various amino acid substitutions of Cys-88 lack ubiquitin-conjugating activity and do not carry out any of the known RAD6 biological functions (Sung et al. 1990, 1991a,b). Thus, ubiquitin conjugation by RAD6 to cellular targets is essential for DNA repair, UV mutagenesis, sporulation, maintenance of normal cell growth, and E3-dependent protein degradation.

The structure and function of RAD6 have been conserved to a remarkable degree among eukaryotes (Reynolds et al. 1990; Koken et al. 1991). RAD6 homologous proteins from the fission yeast Schizosaccharomyces pombe and from humans all share at least $68 \%$ amino acid identity with RAD6. Importantly, when expressed in $S$. cerevisiae, these RAD6 homologs partially complement the UV sensitivity of a rad6 deletion (rad64) mutant and they restore UV-induced mutagenesis in the rad $6 \Delta$ mutant to wild-type level. This result strongly suggests that the structure and function of proteins with which RAD6 interacts and to which RAD6 conjugates ubiquitin are also conserved evolutionarily.

In addition to $R A D 6, R A D 18$, another member of the $R A D 6$ epistasis group, is also required for postreplication repair of UV-damaged DNA (Prakash 1981), and mutations in both of these genes have an equally pronounced effect on UV survival. However, unlike RAD6, UV mutagenesis and sporulation are not affected by mutations in the RAD18 gene (Lawrence 1982; Jones et al. 1988). The rates of spontaneous and UV-induced mitotic recombination are elevated in rad6 and rad18 mutants, suggesting that these genes mediate repair in a nonrecombinational manner (Prakash et al. 1993). Other prominent members of the RAD6 epistasis group are $R A D 5, R E V 1$, and $R E V 3$. Of these, $R A D 5$ functions primarily with $R A D 18$ in error-free postreplication repair (Johnson et al. 1992). The REV1 and REV3 genes are essential for UV mutagenesis; however, they affect UV sensitivity only marginally (Johnson et al. 1992), suggesting that mutagenic replicative bypass of UV lesions constitutes a minor repair pathway. This idea is supported by the observation that mutations in REV3 have no perceptible effect on postreplication repair of UV-damaged DNA (Prakash 1981). Thus, in contrast to RAD6, which affects postreplication repair in both error-free and mutagenic ways, $R A D 18$ and $R A D 5$ mediate predominantly the error-free pathway of repair, and REV1 and REV3 function in the mutagenic pathway.

The RAD6 ubiquitin-conjugating activity could affect DNA repair distal from the site of DNA damage. Alternatively, a mechanism might exist that targets the ubiquitin-conjugating activity of RAD6 directly to the damage sites. In this study we demonstrate that RAD6 physically interacts with the RAD18 protein and provide evidence that complex formation between RAD6 and RAD18 is an obligatory step in DNA repair. We also show that the domain in RAD6 for interaction with RAD18 protein lies outside of that required for interac- tion with the UBR1 protein, the E3 component in $S$. cerevisiae. Whereas RAD6 protein does not bind DNA, RAD18 binds single-stranded DNA (ssDNA) and via complex formation with RAD6, targets the latter to ssDNA. The implications of our findings are discussed.

\section{Results}

\section{Antibodies specific for RAD6 and RAD18}

Antibodies specific for RAD6 and RAD18 were isolated from rabbit antisera by affinity chromatography and covalently coupled to protein A-agarose beads for use in immunoprecipitation experiments (Bailly et al. 1992). After incubation with yeast extract and washing, immunobeads were treated with $1 \%$ SDS to elute bound proteins, which were resolved in denaturing polyacrylamide gels and analyzed by immunoblotting. Anti-RAD6 immunobeads precipitated the $20-\mathrm{kD}$ RAD6 protein (Sung et al. 1990; Fig. 1A). Immunobeads specific for RAD18 precipitated a $66-\mathrm{kD}$ protein that reacted with antiRAD18 antibodies in immunoblot analysis (Fig. 1B). This $66-\mathrm{kD}$ band is the product of $R A D 18 \Delta$ because (1) it was absent in extract of yeast cells harboring the rad18 mutation (Fig. 1B, lane 2) and (2) its level increased with the RAD18 gene dosage (Fig. 2A, top, cf. lanes 5 and 6). The quantity of immunoprecipitable RAD6 is not affected by the rad18 $\Delta$ mutation (Fig. 1A, cf. lanes 2 and 3), and likewise, the rad $6 \Delta$ mutation does not alter the amount of RAD18 protein (Fig. 1B, cf. lanes 1 and 3). Neither RAD6 nor RAD18 was precipitated by protein A-agarose beads bearing antibodies specific for the nu-

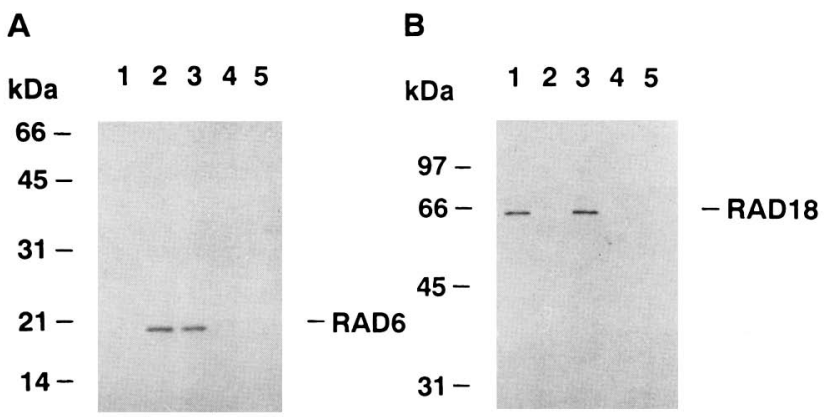

Figure 1. $(A)$ Immunoprecipitation of RAD6 protein. Extracts from the $R A D^{+}$strain LP3041-6D (lane 3) and from its isogenic

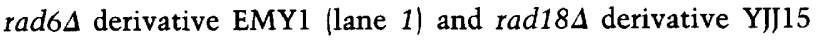
(lane 2) were mixed with protein A-agarose beads bearing antiRAD6 antibodies. The SDS eluates of immunoprecipitates were analyzed by immunoblotting for their content of RAD6 protein. As shown in lanes 4 and 5, respectively, RAD6 is not precipitated from the $R A D^{+}$extract by anti-RADl and anti-RAD10 protein A-agarose immunobeads. $(B)$ Immunoprecipitation of RAD18 protein. The SDS eluates of anti-RAD18 immunoprecipitates were analyzed by immunoblotting for their content of RAD18. Lanes 1-3 contain samples from rad6s, $\operatorname{rad} 18 \Delta$, and $R A D^{+}$extracts, respectively. RAD18 protein in $R A D^{+}$extract is not precipitated by anti-RAD1 (lane 4) and anti-RAD10 (lane 5) immunobeads. 
A

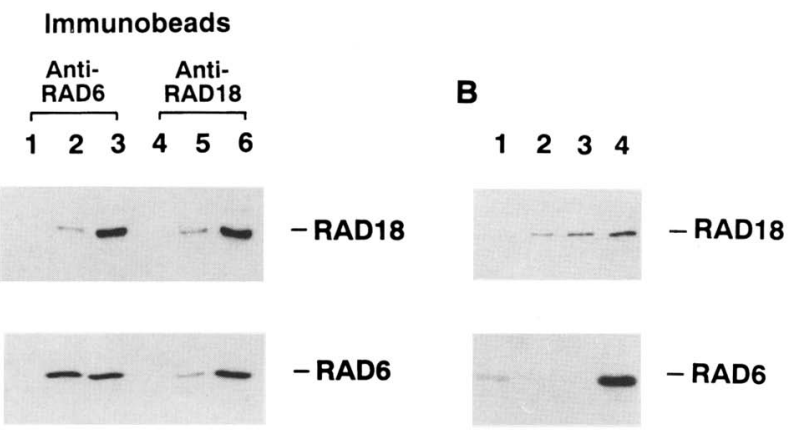

Figure 2. RAD6 and RAD18 proteins are stably associated in vivo. (A) Coimmunoprecipitation of RAD6 and RAD18. Extracts from rad6 $\Delta$ cells (lane 1), rad18 $\Delta$ cells (lane 4), $R A D^{+}$cells (lanes 2,5 ), and $R A D^{+}$cells harboring the $2 \mu$ multicopy RAD18containing plasmid pJJ136 (lanes 3,6 ) were mixed with antiRAD6 immunobeads (lanes 1-3) and anti-RAD18 immunobeads (lanes 4-6). The SDS eluates of immunoprecipitates were analyzed by immunoblotting for their content of RAD6 (bottom) and RAD18 (top). (B) Strength of RAD6/RAD18 interaction. The RAD6/RAD18 complex in $5 \mathrm{ml}$ of extract of $R A D^{+}$cells harboring pJJ136 was immobilized on $50 \mu \mathrm{l}$ of anti-RAD6 immunobeads. The immunocomplex was washed at $25^{\circ} \mathrm{C}$ with $500 \mu \mathrm{l}$ of $1 \mathrm{M} \mathrm{NaCl}$ (lane 1), followed by the same volume of $0.05 \%$ SDS (lane 2), $0.1 \%$ SDS (lane 3), and 1\% SDS (lane 4). The various washes were treated with cold acetone to precipitate protein and analyzed for their content of RAD6 (bottom) and RAD18 (top).

cleotide excision repair proteins RADl and RAD10 (Bailly et al. 1992, Fig. 1A,B, lanes 4,5).

\section{RAD6 and RAD18 exist as a complex in vivo}

To investigate whether the RAD6 and RAD18 proteins interact physically, immunoprecipitation was carried out with anti-RAD6 immunobeads and the SDS eluate examined for the presence of RAD18 protein by immunoblotting, and conversely, the content of RAD6 protein in anti-RAD18 immunoprecipitate was determined. Apparently, a quantitative amount of RAD18 protein was precipitated by anti-RAD6 immunobeads from extract of wild-type cells (Fig. 2A, top, cf. lanes 2 and 5), but no RAD18 was precipitated by anti-RAD6 immunobeads when extract of rad $6 \Delta$ cells was used (Fig. $2 \mathrm{~A}$, top, lane 1). Thus, association of RAD18 with anti-RAD6 immunobeads requires the RAD6 protein, strongly suggesting the existence of a physical complex of the two proteins in wild-type cells. This conclusion is supported by the observation that a sizable proportion $(\sim 10 \%)$ of the RAD6 pool in wild type extract coprecipitated with the RAD18 protein on anti-RAD18 immunobeads (Fig. 2A, bottom, cf. lanes 2 and 5). Introduction into a $R A D^{+}$yeast strain of pIJ136, a multicopy plasmid carrying the RAD18 gene in a yeast $2 \mu$ vector, not only increased the level of immunoprecipitable RADl8 protein by $\sim 10$-fold (Fig. 2A, lane 6) but also the amount of RAD18 in the RAD6/RAD18 complex, apparently to the same extent (Fig. 2A, top, lane 3). These observations indicate that complex formation between RAD6 and RAD18 is a highly efficient process, with RAD18 being the limiting component.

\section{RAD6/RAD18 complex is highly stable}

To examine the strength of interaction, the complex of RAD6 and RAD18 was isolated on anti-RAD6 immunobeads and eluted with $1 \mathrm{M} \mathrm{NaCl}$ followed by increasing concentrations of the protein denaturant SDS $10.05 \%$, $0.1 \%$, and $1 \%$ ). The eluates were subjected to SDS-PAGE and analyzed by immunoblotting for their content of RAD6 and RAD18. Because RAD6 in the immunocomplex was bound by antibodies directly, $1 \%$ SDS was required for its elution (Fig. 2B, bottom, lane 4). Under the conditions stated, examination of RAD18 content in the various washes would yield information concerning the avidity of RAD18 for RAD6. As shown in Figure $2 \mathrm{~B}$ /top, lane 1), the RAD6/RAD18 complex withstood the challenge of $1 \mathrm{M} \mathrm{NaCl}$ and was partially resistant to low concentration of SDS, as only $16 \%$ and $25 \%$ of RAD 18 was eluted by $0.05 \%$ and $0.1 \%$ of the protein denaturant, respectively (Fig. 2B, top, lanes 2,3 ), reflecting a high degree of stability of the protein complex. Similar conclusions concerning the stability of the RAD6/RAD18 complex were drawn when anti-RAD18 immunobeads were used for isolating the complex (data not shown).

\section{RAD6 and RAD18 interact directly}

Coimmunoprecipitation of two proteins can occur via direct interaction between them or through an intermediary. To distinguish between these possibilities, we employed a modified blotting technique to determine whether RAD6 and RAD18 interact directly. The SDS eluates of anti-RAD18 immunoprecipitates from yeast extracts obtained from the rad18 $R A D^{+}$, and $R A D^{+}$ cells carrying the $2 \mu$ multicopy RAD18 plasmid pJJ136, and thus differing in their RAD18 gene dosage, were electrophoresed in a denaturing polyacrylamide gel, followed by transfer of the resolved proteins onto nitrocellulose. The nitrocellulose sheet with increasing amounts of immunopurified RAD18 was incubated in buffer containing bovine serum albumin to saturate protein-binding sites on the filter and to renature RAD18, followed by a second incubation with homogeneous RAD6 protein before being probed with anti-RAD6 antibodies. In the control experiment, a duplicate nitrocellulose filter was treated identically except that RAD6 protein was omitted in the incubation that preceded probing with anti-RAD6 antibodies. As shown in Figure 3, there was no cross-reactivity between renatured RAD18 and antiRAD6 antibodies (Fig. 3, II), but preincubation of nitrocellulose with RAD6 protein rendered the RAD18 band immunoreactive with the anti-RAD6 antibodies (Fig. 3, III), indicating binding of RAD6 by the immobilized RAD18 protein. Thus, complex formation between RAD6 and RAD18 can occur without an intermediary. 
Bailly et al.

\section{3}

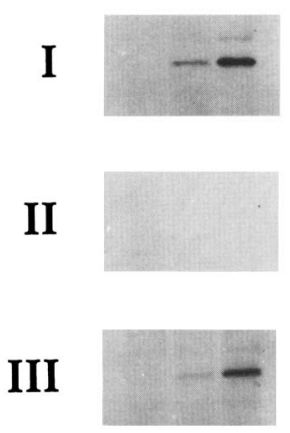

Figure 3. RAD6 and RAD18 interact directly. Nitrocellulose blot containing SDS eluates of anti-RAD18 immunoprecipitates from extracts of rad18 $\triangle$ cells (lane 1), $R A D^{+}$cells (lane 2), and $R A D^{+}$cells harboring the $2 \mu$ RAD18 plasmid pJ)136 (lane 3) was probed with anti-RAD18 antibodies $(I)$, anti-RAD6 antibodies $\{I I\}$, or first incubated with homogeneous RAD6 protein before being probed with anti-RAD6 antibodies $(I I I)$. The same region of the nitrocellulose blot is shown in $I, I I$, and $I I I$, and these blots were obtained from transfer of three separate sets of SDS eluates of anti-RAD18 immunoprecipitates.

\section{RAD6 associates with RAD18 and with UBR1 in separate complexes}

The RAD6 ubiquitin-conjugating function mediates degradation of proteins bearing destabilizing amino-terminal residues (Dohmen et al. 1991; Sung et al. 1991a; Watkins et al. 1993) in the amino-end rule-dependent proteolytic pathway. This mode of protein degradation also requires the $U B R 1$-encoded $\mathrm{E} 3$ protein, which provides the binding site for the amino terminus of proteolytic substrates. UBRl protein physically interacts with RAD6, bringing RAD6 to attach multiple molecules of ubiquitin to a lysine residue in the bound substrates (Dohmen et al. 1991; Sung et al. 199la; Watkins et al. 1993).

In view of our present finding that RAD6 and RAD18 are associated in a complex, it was of considerable interest to determine whether RAD6 associates with RAD18 and UBR1 simultaneously to form a three-component complex. To address this, the $R A D^{+}$yeast strain

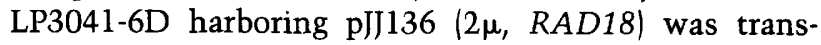
formed with the plasmid pSOB44 $(2 \mu, A D C 1-U B R 1 / h a)$, which contains the $U B R 1$ gene tagged with a carboxyterminal 9-residue extension derived from hemagglutinin (HA) of influenza virus under the control of the $A D C 1$ promoter (Dohmen et al. 1991). Cell extract from LP3041-6D (pJJ136 and pSOB44) was treated with immunobeads specific for RAD6 and RAD18, and also with immunobeads bearing the monoclonal antibody 12CA5 (Field et al. 1988) specific for the HA epitope in UBR1/ HA. The SDS eluates of the various immunoprecipitates were then examined by immunoblotting for their content of the three proteins to determine the hierarchical order of interaction. As expected, both RAD18 and
UBR1/ha coprecipitated with RAD6 protein (Fig. 4A, I, II, and III, lane 1). In the anti-RAD18 and anti-UBR1/ha immunoprecipitations, we observed the coprecipitation of RAD6 with RAD18 (Fig. 4A, I and II, lane 2) and of RAD6 with UBR1/ha (Fig. 4A, I and III, lane 3) but did not detect any association between RADl8 and UBR1/ ha (Fig. 4A, II and III, lanes 2 and 3). Two other independent experiments gave similar results. Thus, it appears that RAD6 forms separate complexes with RAD18 and with UBR1. The possibility of formation of a small amount of ternary complex involving the three proteins, however, cannot be excluded entirely.

\section{The evolutionarily conserved RAD6 amino terminus is not involved in interaction with RAD18}

The amino terminus of RAD6 represents the most conserved segment from yeast to humans (Watkins et al. 1993). To determine the biological role of the RAD6 amino terminus, we deleted the first 9 amino acid residues in RAD6 (Watkins et al. 1993). The rad6 ${ }_{\Delta 1-9}$ mutation engenders a defect in amino-end rule protein degradation and in sporulation, and it affects the proficiency of DNA repair. These phenotypic manifestations suggested that the RAD6 amino terminus may be involved in protein-protein interactions in the various biological functions of RAD6. Consistent with this idea, we showed previously that the failure of the rad6 $6_{\Delta 1-9}$ protein to mediate amino-end rule protein degradation is

A

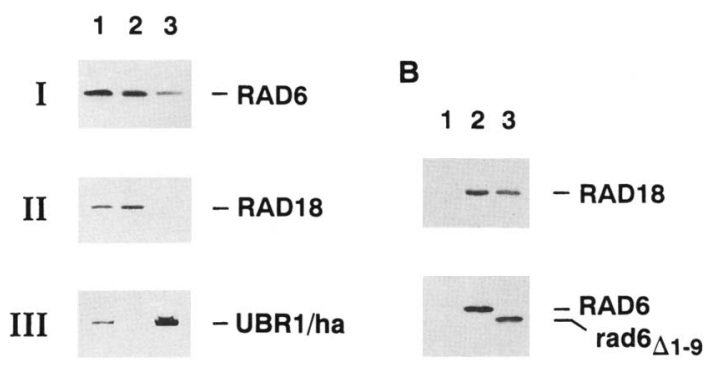

Figure 4. RAD6 complexes with RAD18 and with UBR1 protein separately. (A) Distinct complexes of RAD6/RAD18 and RAD6/UBR1. Extract from $R A D^{+}$cells harboring both pJJ136 $(2 \mu, R A D 18)$ and pSOB44 $(2 \mu, A D C 1-U B R 1 / h a)$ was subjected to immunoprecipitation with anti-RAD6 immunobeads (lane 1), anti-RAD18 immunobeads (lane 2), and immunobeads bearing the monoclonal antibody $12 \mathrm{CA} 5$ specific for the ha epitope in UBRI/ha (lane 3!. The SDS eluates of immunoprecipitates were analyzed for their content of RAD6 (I), RAD18 (II), and UBR/ha $(I I I) .(B)$ The highly conserved RAD6 amino terminus is not required for RAD6/RAD18 interaction. Extract $(3 \mathrm{ml})$ from rad $6 \Delta$ cells harboring pJJ136 was subjected to immunoprecipitation with anti-RAD6 immunobeads (lane 1). The same extract was incubated with $1 \mu \mathrm{g}$ of purified RAD6 protein (lane 2) or purified rad $6_{\Delta 1-9}$ protein (lane 3 ) at $4^{\circ} \mathrm{C}$ for $2 \mathrm{hr}$ before being subjected to anti-RAD6 immunoprecipitation. The SDS eluates of immunoprecipitates were analyzed for their RAD6 or rad6 $_{\Delta 1-9}$ protein content (bottom) and RAD18 content (top). 
attributable to the inability of the mutant protein to interact with the UBR1 protein (Watkins et al. 1993).

Here, we examine whether the rad6 ${ }_{\Delta 1-9}$ mutant protein can interact with RAD18 protein using the same experimental conditions where $\mathrm{rad}_{\Delta 1-9}$ protein fails to complex with UBR1 (Watkins et al. 1993). The RAD6 protein or the rad6 ${ }_{\Delta 1-9}$ protein, both purified to homogeneity as described (Sung et al. 1991a; Watkins et al. 1993) were added to extract from rad6 $\Delta$ cells harboring the $2 \mu R A D 18$ plasmid pJJ136. After incubation on ice, the extract was subjected to immunoprecipitation with anti-RAD6 immunobeads. As shown in Figure 4B, a similar amount of RAD18 protein coprecipitated with RAD6 and rad6 $_{\Delta 1-9}$ proteins (lanes 2,3), indicating that the amino terminus of RAD6 protein is dispensable for complex formation with RAD18.

Semidominance of overproduction of $\operatorname{rad} 6$ Ala-88 mutant protein suggests biological significance of physical interaction

Previously, we evaluated the biological role of the RAD6 ubiquitin-conjugating function by mutating Cys- 88 in RAD6, the site of thioester formation with ubiquitin during its conjugation to substrates (Sung et al. 1990; Hershko 1991), to other residues, including alanine. The protein encoded by the rad 6 Ala- 88 allele, which resembles the rad6 6 mutant in UV sensitivity, is devoid of ubiquitin-conjugating activity (Sung et al. 1990). In this study we found that the same amount of RADl 8 can be coprecipitated from cell extract with rad6 Ala-88 mutant protein as with wild-type RAD6 protein by anti-RAD6 immunobeads (Fig. 5A, cf. lanes 2 and 3), indicating that rad6 Ala-88 protein is as efficient as the RAD6 protein in interacting with RAD18.

Interestingly, a fiftyfold overexpression of rad 6 Ala- 88 protein by use of the $A D C I$ promoter in plasmid pR648 (Sung et al. 1990) in the $R A D^{+}$strain renders cells highly sensitive to UV light (Fig. 5B). For example, at the relatively low UV dose of $10 \mathrm{~J} / \mathrm{m}^{2}$, there was only $\sim 1 \%$ survival of $R A D^{+}$cells harboring pR648, whereas essentially no increase in UV sensitivity is observed in the $R A D^{+}$cells carrying the $A D C I$ vector $\mathrm{pSCW} 231$, which lacks the rad6 Ala-88 insert present in pR648; rad64 cells showed a $0.1 \%$ survival at this UV dose. The most plausible explanation of this phenomenon is that the excess of rad $6 \mathrm{Ala}-88$ protein sequesters RAD18 protein, thereby compromising the cellular ability to repair UVdamaged DNA by limiting the availability of cellular RAD18 protein for complex formation with RAD6 protein. Consistent with this interpretation, increasing the $R A D 18$ gene dosage by the introduction of the multicopy $2 \mu$ RAD18 plasmid pJJ136, which overproduces RAD18 protein $\sim 10$-fold (Fig. $2 \mathrm{~A}$ ), largely overcame the observed semidominance of rad6 Ala-88, resulting in restoration of almost wild-type level of resistance to UV light (Fig. $5 \mathrm{~B}$ ). On the basis of these observations, it may be argued that the functional integrity of postreplication repair requires that the ubiquitin-conjugating activity of RAD6 be physically associated with RAD18.

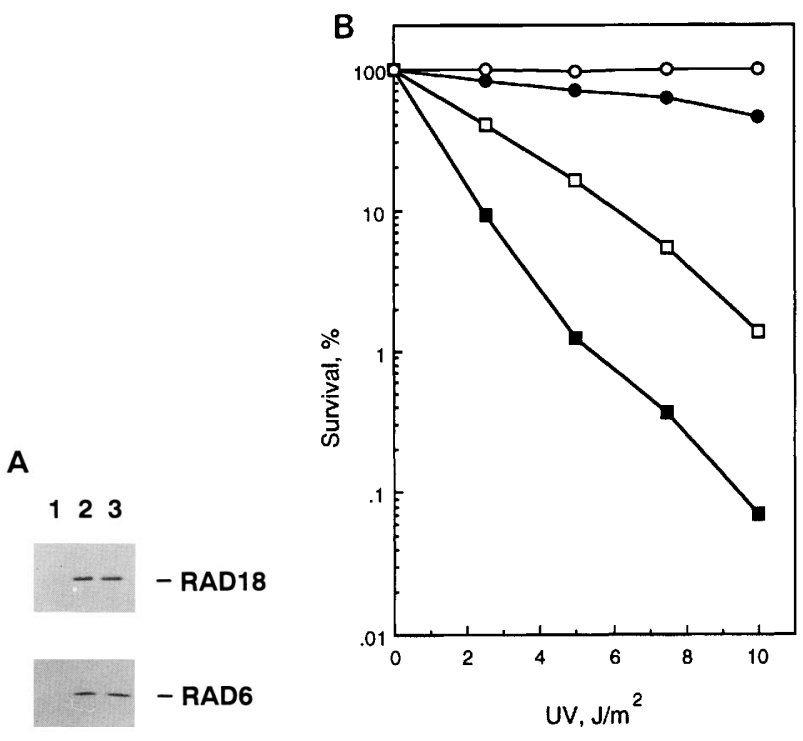

Figure 5. Interaction of $\mathrm{rad} 6 \mathrm{Ala}-88$ protein with RAD18. $(A)$ Coimmunoprecipitation of rad6 Ala-88 and RAD18. Extract of rad $6 \Delta$ cells harboring the low-copy $R A D 6$ plasmid pR67 (lane 2) or the low copy rad6 Ala-88 plasmid pR647 (lane 3) was subjected to immunoprecipitation with anti-RAD6 immunobeads. In lane 1, extract of rad6 $\Delta$ cells was subjected to the same immunoprecipitation treatment. The SDS eluates of immunoprecipitates were analyzed for RAD6 (or rad6) and RAD18 content. (B) Overexpression of rad6 Ala-88 protein confers UV sensitivity to $R A D^{+}$strain. UV survival of $\operatorname{rad} 6 \Delta(\square), R A D^{+}$harboring the $A D C 1$ vector pSCW231 $(O), R A D^{+}$harboring the $A D C 1-$ rad6 Ala-88 plasmid pR648 and the $2 \mu$ RAD18 plasmid pJJ136 (O), and $R A D^{+}$harboring pR648 and the $2 \mu$ vector pTB220, which lacks the $R A D 18$ gene ( $\square$ ). All strains are isogenic derivatives of the $R A D^{+}$strain YRP10.

\section{RAD18 endows complex with DNA-binding ability}

Because of their involvement in a major pathway of DNA repair, we investigated whether RAD6 and RAD18 proteins have DNA-binding activity using the Southwestern technique (Bowen et al. 1980; Brill and Stillman 1989 ). In this assay RAD6 protein (25 and 50 pmoles; Sung et al. 1988) and immunopurified RAD18 protein $(9$ and 18 pmoles) were electrophoresed in denaturing polyacrylamide gels, transferred onto nitrocellulose, and, after renaturing treatment, incubated with ${ }^{32} \mathrm{P}$-labeled linear double-stranded (ds) M13 DNA or ss M13 DNA generated from the double-stranded form by thermal denaturation. After extensive washing, the nitrocellulose filters were subjected to autoradiography to detect DNA binding. This analysis revealed that RAD6 does not interact with either dsDNA (data not shown) or ssDNA (Fig. 6A, bottom), whereas RAD18 binds ssDNA (Fig. 6A, top) but not the double-stranded form (data not shown).

Results from the Southwestern analysis suggest that complex formation with RAD18 protein may confer upon RAD6 the ability to interact with ssDNA. To verify this, we carried out the following experiments. Cell extract from the rad18 $\Delta$ strain YJJ15 was applied onto a 
A

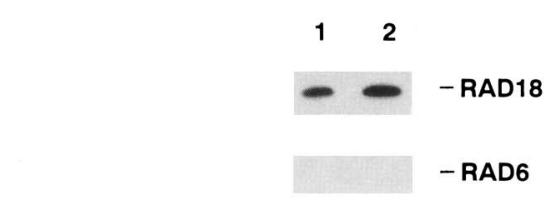

B

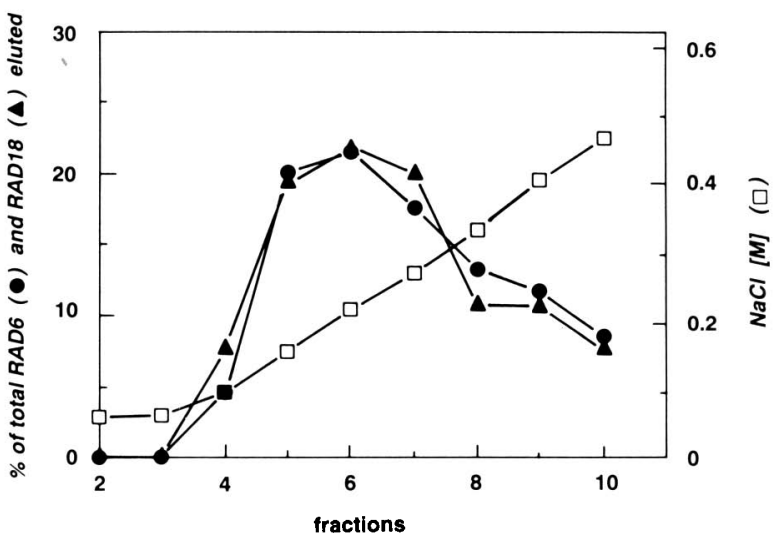

Figure 6. RAD18 is the DNA-binding component in RAD6/ RAD18 complex. $(A)$ Southwestern DNA binding analysis. Nitrocellulose bound purified RAD6 protein $[25$ and 50 pmoles in lanes 1 and 2, respectively (bottom)] and immunopurified RADl 8 protein $[9$ and 18 pmoles in lanes 1 and 2 , respectively (top)] were incubated with ${ }^{32} \mathrm{P}$-labeled ssDNA and subjected to autoradiography to detect DNA binding, as described in Materials and methods. $(B)$ RAD6/RAD18 complex is retained on ssDNA agarose. Extracts from $R A D^{+}$cells harboring the $2 \mu$, RAD18 plasmid pJI136 were passed through ssDNA-agarose, and column gradient fractions analyzed for their content of RAD6 and RAD18 proteins by immunoblotting. The immunoblots were scanned by densitometry to determine the relative amounts of RAD6 $(O)$ and $\operatorname{RAD} 18(\mathbf{A})$ in the ssDNA-agarose column fractions for a graphic representation of the results. (ㅁ) $\mathrm{NaCl}$ concentration in the fractions.

column of ssDNA-agarose. After washing, the column was eluted with a $5 \mathrm{ml} \mathrm{NaCl}$ gradient from 50 to $750 \mathrm{mM}$, and fractions $(0.5 \mathrm{ml}$ each) were analyzed by immunoblotting for their content of RAD6 protein. RAD6 does not bind to ssDNA-agarose under the conditions used (data not shown). In contrast, we found that a sizable proportion of the RAD6 pool in extract of YTJ15 harboring $\mathrm{pJ} 136(2 \mu R A D 18)$ was retained on ssDNA-agarose and the bound RAD6 protein eluted as a broad peak from 100 to $300 \mathrm{~mm} \mathrm{NaCl}$ (Fig. 6B). Determination of the RAD18 content of the gradient fractions showed a constant ratio of RAD6 to RAD18 across the entire gradient (Fig. 6B), providing strong evidence that RAD6 was brought onto ssDNA by RAD18 protein. These observations strongly implicate RAD18 as the DNA-binding component of the RAD6/RAD18 complex.

RAD6 homologs from S. pombe and humans also interact with RAD18

The RAD6 structure and function have been conserved to a remarkable degree throughout eukaryotic evolution. The protein encoded by the $r h p 6^{+}$gene of the fission yeast $S$. pombe shows strong homology $177 \%$ identical amino acid residues) to RAD6 protein, and a deletion mutation of $r$ hp6 ${ }^{+}$from the genome renders cells highly sensitive to UV light (Reynolds et al. 1990). Two RAD6 homologs, HHR6A and HHR6B, have been identified in humans; the protein products of these genes share $\sim 70 \%$ identical residues with the RAD6 protein (Koken et al. 1991).

Because the $r h p 6^{+}, H H R 6 A$, and HHR6B genes partially complement the UV sensitivity of the rad $6 \Delta \mathrm{mu}$ tant (Reynolds et al. 1990; Koken et al. 1991), we examined whether their protein products interact with RAD18 protein. To determine this, we introduced the $S$. pombe and human RAD6 homologs, which were expressed in $S$. cerevisiae utilizing the $A D C I$ promoter, into the rad6 $\Delta$ strain harboring the plasmid pJJ136 $(2 \mu$ $R A D 18$ ), and subjected cell extracts to immunoprecipitation. As a control, we included in the immunoblot analysis the SDS eluate of anti-RAD6 immunobeads after incubation with wild-type extract (Fig. 7, lane 2). The anti-RAD6 immunobeads recognized the various RAD6 homologous proteins and coprecipitated the RAD18 protein in all cases, indicating an ability of these RAD6 homologous proteins to complex with RAD18 (Fig. 7). None of the RAD6 homologous proteins, however, interacts with RAD18 as efficiently as does RAD6 (Fig. 7), which could account for the reason that these RAD6 homologs are only partially effective in substituting for RAD6 (Reynolds et al. 1990; Koken et al. 1991).

\section{Discussion}

\section{A specific complex of RAD6 and RAD18 proteins}

Here, we demonstrate that the majority of the RAD 18 protein in wild-type cells can be coimmunoprecipitated with RAD6 protein, and likewise, a sizable portion of the cellular RAD6 pool can be coimmunoprecipitated with RAD18 protein. Introduction into yeast cells of a multicopy plasmid bearing the RAD18 gene increases the

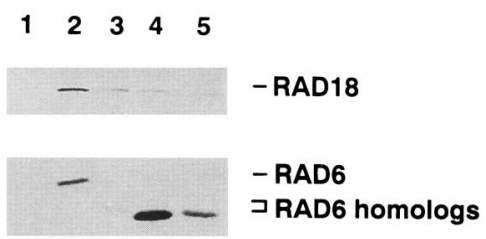

Figure 7. RAD6 homologs from $S$. pombe and humans interact with RAD18. Extracts from rad6 $\Delta$ cells harboring $2 \mu, R A D 18$ plasmid pJI136 (lane 1), pJJ136 and the $A D C 1-r h p 6^{+}$plasmid pRR429 (lane 3), pJl136 and the ADC1-HHR6A plasmid pRR510 (lane 4), and pJJ136 and the ADC1-HHR6B plasmid pRR518 (lane 5) were subjected to anti-RAD6 immunoprecipitation. The SDS eluates of immunoprecipitates were analyzed for their content of RAD6 homologs (bottom) and of RAD18 (top). As control, extract of $S$. cerevisiae $R A D^{+}$cells (lane 2) was used. 
amount of immunoprecipitable RAD6/RAD18 complex to an extent proportional to the overproduction of RAD18 protein. From these observations, we conclude that RAD6 and RAD18 proteins form a specific complex.

The complex of RAD6 and RAD18 cannot be disrupted by $1 \mathrm{M} \mathrm{NaCl}$ and is partially resistant to low concentrations of SDS, indicating a high degree of stability of the complex. We showed that RAD18 protein immunopurified from cell extract, after being resolved in a denaturing polyacrylamide gel and transferred onto nitrocellulose, physically interacts with RAD6 protein. This observation indicates that complex formation between RAD6 and RAD18 proteins can occur in the absence of a protein intermediary or a cofactor such as nucleic acid or a nucleoside triphosphate.

Consistent with the ability of $S$. pombe and human $R A D 6$ homologs to partially complement the UV sensitivity of rad6 mutants (Reynolds et al. 1990; Koken et al. 1991), we find that the RAD6 homologous proteins from $S$. pombe and humans also physically interact with the RAD18 protein. Our results predict that the as yet unidentified RAD18 homolog from other eukaryotes also forms a specific complex with its respective RAD6 homolog in accomplishing postreplication repair.

\section{Biological implications of RAD6/RAD18 complex formation}

A 50-fold overproduction of the biologically inactive rad6 Ala-88 protein in $R A D^{+}$cells imparts a high degree of UV sensitivity, which can be corrected by concomitant overexpression of $R A D 18$. Overproduction of rad6 Ala-88 protein in wild-type cells also causes an inhibition of the amino-end rule pathway (Madura et al. 1993). Because rad6 Ala- 88 protein retains the ability to interact with RAD18, a plausible explanation for the semidominance of rad6 Ala-88 in wild-type cells is the titration of RAD1 8 by the mutant rad6 protein. These observations suggest that complex formation between RAD6 and RAD18 is an obligatory step in the process of postreplication repair.

RAD18 protein exhibits a ssDNA-binding activity in Southwestern analysis and it is retained on ssDNA-agarose. These findings contrast with the negative result from the Southwestern DNA-binding analysis for RAD6 and the experiment that demonstrated the inability of RAD6 alone to bind ssDNA-agarose. In DNA-binding studies utilizing nitrocellulose filters, under a wide range of $\mathrm{pH}$ and $\mathrm{KCl}$ concentrations, we also have not detected any interaction between purified RAD6 and ssDNA and dsDNA (P. Sung, unpubl.). Taken together, our results indicate that RAD6 does not bind DNA, whereas RAD18 has the capacity of binding at least ssDNA. Importantly, we found that the RAD6/RAD18 complex in cell extract is retained on ssDNA-agarose, suggesting that RAD6 protein can be targeted to ssDNA via its interaction with RAD18 protein.

DNA polymerases pause at sites of DNA damage because of their inability to efficiently bypass DNA lesions. Therefore, during replication of DNA damaged by
UV light, gaps are created in the newly synthesized strand across from the damage site. The single-stranded regions that result from the stalling of DNA polymerases could be specifically recognized and bound by the RAD6/RAD18 complex, and, subsequently, RAD6 may mediate the degradation of the DNA polymerase subunits and/or associated protein factors via ubiquitin conjugation to these components. Turnover of specific proteins from the replication complex stalled at the damage site could then allow access of DNA repair proteins such as RAD5, RAD18, and perhaps others to the replication complex. The modified replication complex could then fill the damage-associated gap in the nascent DNA strand by a "copy choice" replication mechanism, utilizing the intact duplex sister chromatid for template information. We expect this process of postreplication gap filling to be relatively error free.

\section{Targeting of RAD6 ubiquitin-conjugating activity}

In addition to its requirement in DNA repair, RAD6 protein functions in the ubiquitin-dependent amino-end rule protein degradation pathway (Dohmen et al. 1991; Sung et al. 1991a). The ubiquitin-conjugating activity of RAD6 is targeted to the amino-end rule protein substrates via its interaction with the $\mathrm{E} 3$ protein. Here, we have demonstrated that RAD6 also interacts with the RAD18 protein, provided genetic evidence that this interaction is important for DNA repair, and suggested that RAD18 serves to target the RAD6 ubiquitin-conjugating activity to damage-containing ssDNA regions. We also show that the protein encoded by the rad $6_{\Delta 1-9}$ allele, missing the first 9 residues, and known to be defective in interaction with the $S$. cerevisiae UBR1 gene product, the E3 component of the amino-end rule proteolytic pathway (Watkins et al. 1993), is as proficient as RAD6 in interacting with the RAD18 protein. The highly conserved amino terminus of RAD6 thus seems to be specific for interaction with the UBR1 protein, indicating that a separate domain in RAD6 mediates its interaction with the RAD18 protein.

In summary, RAD6 forms separate complexes with RAD18 and with UBR1, and complex formation with these proteins targets RAD6 to DNA and to proteolytic substrates, thereby allowing RAD6 to effect DNA repair and amino-end rule-dependent protein degradation (Fig. $8)$.

\section{Materials and methods}

Buffers

Buffer A was $50 \mathrm{~mm}$ Tris- $\mathrm{HCl}(\mathrm{pH} 7.5)$, containing $50 \mathrm{~mm} \mathrm{NaCl}$, $0.2 \%$ Triton X-100, 0.1 mM PMSF, $0.5 \mathrm{mM} \mathrm{DTT}$, and $20 \mu \mathrm{g} / \mathrm{ml}$ of aprotinin, chymostatin, leupeptin, and pepstatin A. Buffer B was $50 \mathrm{~mm}$ Tris $-\mathrm{HCl}(\mathrm{pH} 7.5)$, containing $600 \mathrm{mM} \mathrm{KCl}, 10 \mathrm{mM}$ EDTA, $10 \mathrm{~mm}$ 2-mercaptoethanol, 10\% sucrose, and $0.2 \%$ (vol/ vol) Tween 20 . Buffer $\mathrm{C}$ was $25 \mathrm{mM}$ Tris- $\mathrm{HCl}\{\mathrm{pH} 7.0\}$, containing $2 \mathrm{mg} / \mathrm{ml}$ of BSA and $1 \mathrm{~mm}$ DTT. Buffer D was $20 \mathrm{~mm}$ Tris$\mathrm{HCl}$ (pH 7.0), containing $30 \mathrm{~mm} \mathrm{KCl}, 200 \mu \mathrm{g} / \mathrm{ml}$ of BSA, $4 \mathrm{~mm}$ $\mathrm{MgCl}_{2}$, and $1 \mathrm{mM} \mathrm{DTT}$. Phosphate-buffered saline (PBS) was 20 


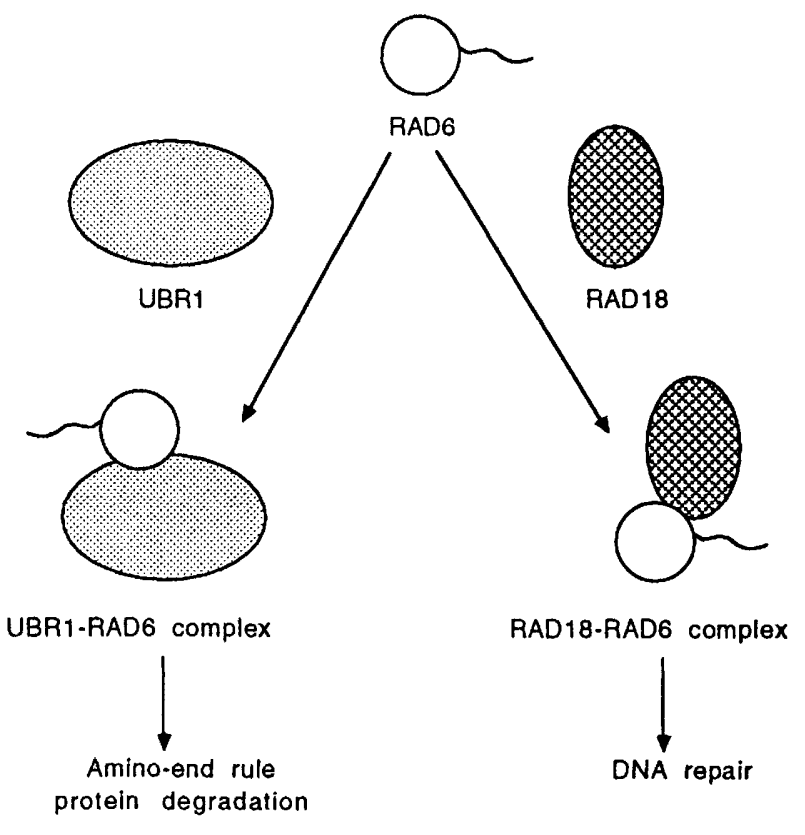

Figure 8. Formation of separate complexes of RAD6 with the UBR1 protein and the RAD18 protein. The UBR1-RAD6 complex targets the RAD6 ubiquitin-conjugating activity to the amino-end rule protein substrates, whereas the RAD18-RAD6 complex targets RAD6 to damage containing ssDNA regions. Degradation of components of the DNA replication machinery stalled at the sites of DNA damage mediated by the ubiquitinconjugating activity of RAD6 is suggested to be necessary for postreplication repair to occur. (Open circles) RAD6 with the polyacidic tail; (stippled ovals) UBR1; (crosshatched ovals) RAD18.

$\mathrm{mM} \mathrm{KH} \mathrm{PO}_{4}$ (pH 7.2), containing $150 \mathrm{~mm} \mathrm{NaCl}$. Proteins were eluted from immunoprecipitates with $1 \%$ SDS in $10 \mathrm{~mm}$ Tris$\mathrm{HCl}$ (pH 7.0).

\section{Yeast strains}

LP3041-6D (MATa RAD ${ }^{+}$leu2-3 leu2-112 trp1s ura3-52). YRP10 (MAT $\alpha$ ade5 his7 leu2 lys1 met14 trp1s ura3). Strains

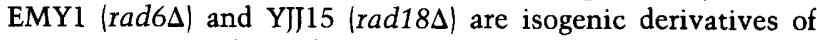
LP3041-6D, in which the LEU2 gene replaces the RAD6 and RAD18 genes, respectively.

\section{Plasmids for protein expression}

pR67 (RAD6) and pR647 (rad6 Ala-88) are low-copy centromeric yeast plasmids containing the URA3 marker. pIJ 136 is a multicopy yeast plasmid containing the $2 \mu$ plasmid replication origin, the URA3 marker, and RAD18 gene under the control of its own promoter. pSCW231 (Sung et al. 1987) is a $2 \mu$-based vector containing the TRP1 marker and the yeast $A D C I$ promoter for protein expression. pSCW242 (ADCI-RAD6), pR648 (ADCIrad6 Ala-88), pRR429 (ADCI-rhp6 ${ }^{+}$), pRR510 (ADCI-HHR6A), pRR518 (ADCI-HHR6B), and pR661 (ADCI-rad6 $41-9)$ are all derived from pSCW231. pSOB44 (Dohmen et al. 1991) is a 2 $\mu$-based plasmid that contains the TRP1 marker and the UBR1/ ha gene under the control of the $A D C 1$ promoter. pJJ309 (tac$R A D 18$ ) contains the $R A D 18$ gene under the control of the Escherichia coli tac promoter.
Protein purification

RAD6 protein was purified from yeast strain CMY135 harboring pSCW242 (ADCI-RAD6) as described previously (Sung et al. 1988). The rad6 ${ }_{\Delta 1-9}$ mutant protein was purified from the rad6 $\Delta$ strain EMY1 harboring pR661 as described (Watkins et al. 1993). To purify RAD18 protein for use as an immunogen, an exponential culture of E. coli JM101 harboring pJJ309 (tac-RAD18) was treated with $2 \mathrm{mM}$ IPTG for $4 \mathrm{hr}$ to induce synthesis of RAD18. Cells were ruptured in buffer B using a French press, and the particulate material was collected, suspended in SDS buffer (Laemmli 1970) at $10 \mathrm{ml}$ per liter original culture, and boiled for $5 \mathrm{~min}$ to solubilize proteins. After centrifugation, 2 $\mathrm{ml}$ of the clear supernatant containing $\sim 1 \mathrm{mg}$ of $\mathrm{RADl} 8$ in $\sim 5$ $\mathrm{mg}$ of total protein was fractionated in a $3-\mathrm{mm}$-thick denaturing $10 \%$ polyacrylamide gel; RADl8 protein was eluted from gel strips with the aid of an Elutrap (Schleicher \& Schuell). Purified RAD 18 protein was dialyzed against PBS $\left(10 \mathrm{mM} \mathrm{NaH} \mathrm{PO}_{4}\right.$ at $\mathrm{pH} 7.2$, containing $150 \mathrm{~mm} \mathrm{NaCl}$.

\section{Antibodies}

For antibody production, RAD6 or RAD1 8 protein $(100 \mu \mathrm{g}$ in 0.5 ml) was mixed with an equal volume of Freund's complete adjuvant and injected subdermally at four sites on the back of a rabbit. Boosters containing the same quantity of immunogen with Freund's incomplete adjuvant were administered at biweekly intervals; sera obtained subsequent to the second booster were used as the source of antibodies. To prepare affinity matrix for purification of antibodies, $3 \mathrm{mg}$ of RAD immunogen was covalently attached to $4 \mathrm{ml}$ of cyanogen bromideactivated Sepharose following the instructions of the manufacturer (Pharmacia) and packed into a glass column with internal diameter of $1 \mathrm{~cm}$. Sera were diluted with 2 volumes of PBS and passed several times at room temperature through the affinity column by gravity. The column was washed with 20 volumes of double-strength PBS containing $0.1 \%$ (vol/vol) Tween 20 , and the bound antibodies were eluted with $100 \mathrm{~mm}$ diethylamine (pH 11.5$) ; 1 \mathrm{ml}$ fractions were collected and neutralized immediately with $0.5 \mathrm{ml}$ of $0.5 \mathrm{M}$ Tris- $\mathrm{HCl}(\mathrm{pH} 7.0)$. Fractions possessing $\mathrm{OD}_{280}>0.1$ were pooled, concentrated to a small volume using Centricon-30 (Amicon), and dialyzed against PBS. Ten $\mathrm{OD}_{280}$ units of affinity purified antibodies or nonimmune IgG (Sigma) were cross-linked to $1 \mathrm{ml}$ of protein A-affigel (BioRad) using dimethylpimemilidate (Harlow and Lane 1988) for use in immunoprecipitation experiments. Immunobeads specific for various RAD proteins were stored at $4^{\circ} \mathrm{C}$ in PBS. After elution of antibodies, the affinity matrix was neutralized with $0.1 \mathrm{M}$ Tris- $\mathrm{HCl}(\mathrm{pH} 7.0)$ and retained for additional usage.

\section{Immunoprecipitation}

Yeast cells were cultured in synthetic media and harvested by centrifugation when the titer reached $2 \times 10^{7}$ cells $/ \mathrm{ml}$. To prepare extract, cells were suspended in buffer $\mathrm{A}$ at $3 \mathrm{ml} / \mathrm{g}$ and ruptured by agitating rigorously with $0.5-\mathrm{mm}$ glass beads $(1.5$ gram of beads per gram of $\mathrm{g}$ cells/ using eight $30-\mathrm{sec}$ pulses with intermittent chilling on ice. The cell lysate was clarified by centrifugation $(10,000 \mathrm{~g}$ for $10 \mathrm{~min})$, and $1.5 \mathrm{ml}$ of the supernatant was mixed with $15 \mu \mathrm{l}$ of immunobeads for $60 \mathrm{~min}$ at $25^{\circ} \mathrm{C}$. After three washes with $300 \mu$ l of buffer A, bound proteins were eluted from immunobeads with $300 \mu \mathrm{l}$ of $1 \%$ SDS, precipitated with 3 volumes of acetone $\left(12 \mathrm{hr}\right.$ at $\left.-20^{\circ} \mathrm{C}\right)$, and redissolved in $20 \mu \mathrm{l}$ of SDS sample buffer, and an aliquot (usually $10 \mu \mathrm{l}$ ) was subjected to SDS-PAGE followed by immunoblotting analysis. 


\section{Gel electrophoresis and immunoblotting}

SDS-PAGE was carried out according to Laemmli (1970); $9 \%$ and $12 \%$ gels were used for RAD18 and RAD6, respectively. After electrophoresis, proteins were electroblotted onto nitrocellulose sheets (Schleicher \& Schuell), which were probed with $1 / 1000$ dilution of affinity purified antibodies $\left(O D_{280}=3\right)$ and developed using the indirect peroxidase procedure of Towbin et al. (1979).

\section{RAD6/RAD18 complex formation on nitrocellulose blot}

Nitrocellulose blot containing immunopurified RAD18 protein was soaked at $25^{\circ} \mathrm{C}$ for $1 \mathrm{hr}$ in $10 \mathrm{ml}$ of PBS containing $15 \mathrm{mg} / \mathrm{ml}$ of BSA, $2 \mathrm{mM} 2$-mercaptoethanol, and $0.05 \%$ (vol/vol) Tween 20 before being incubated in the same buffer with $5 \mu \mathrm{g} / \mathrm{ml}$ of RAD6 for $2 \mathrm{hr}$. After washing with $3 \times 10 \mathrm{ml}$ aliquots of buffer, the blot was subjected to probing with anti-RAD6 antibodies.

\section{Southwestern DNA-binding analysis}

To obtain RAD18 protein for the Southwestern analysis, extract was prepared in buffer A from $10 \mathrm{~g}$ of strain LP3041-6D harboring $\mathrm{pJ}) 136(2 \mu R A D 18)$ using a French press. The clear supernatant, obtained after high speed centrifugation $(100,000 \mathrm{~g}$ for 60 min), was applied at $25^{\circ} \mathrm{C}$ onto a 1-ml column of protein A-agarose beads containing cross-linked anti-RAD1 8 antibodies. The column was washed extensively with buffer A before being eluted with $1 \%$ SDS; the protein pool (containing $5 \mu \mathrm{g}$ of $25 \%$ pure RAD18) was concentrated to $100 \mu$ l using Centricon- 30 . Bglli-linearized DNA was treated with calf thymus alkaline phosphatase to remove preexisting $5^{\prime}$ phosphate and labeled with the T4 polynucleotide kinase and $\left[\gamma^{-32} \mathrm{P}\right] \mathrm{ATP}$ to a specific radioactivity of $6 \times 10^{5} \mathrm{cpm} / \mu \mathrm{g}$, and purified by gel filtration using Sephadex G50. To generate ssDNA, the labeled doublestranded form was boiled in $10 \mathrm{~mm}$ Tris- $\mathrm{HCl}$ at $\mathrm{pH} 7.5$ for $3 \mathrm{~min}$ and chilled immediately on ice.

Purified RAD6 and RAD18 proteins $(0.5$ and $1 \mu \mathrm{g})$ were electrophoresed in denaturing polyacrylamide gels and transferred onto nitrocellulose, which was soaked at $4^{\circ} \mathrm{C}$ for $36 \mathrm{hr}$ in an excess of buffer $\mathrm{C}$ to block binding sites on nitrocellulose and to renature RAD6 and RAD18 proteins. The filters were rinsed twice with buffer $\mathrm{D}$ and incubated at $25^{\circ} \mathrm{C}$ for $60 \mathrm{~min}$ in $10 \mathrm{ml}$ of buffer D containing $1.5 \mu \mathrm{g}$ of ${ }^{32} \mathrm{P}$-labeled DNA. After being washed with $6 \times 10 \mathrm{ml}$ buffer $\mathrm{D}$ for a total of $20 \mathrm{~min}$, the filters were blot-dried with Whatman $3 \mathrm{M}$ paper and subjected to autoradiography (2-4 hr exposure) using Kodak XRP films.

\section{ssDNA-agarose chromatography}

Cell extract was prepared using a French Press from 3 grams of the rad18 $\Delta$ yeast strain YJI15 or the $R A D^{+}$strain LP3041-6D harboring pI) $136(2 \mu R A D 18)$ in $5 \mathrm{ml}$ of buffer A. After centrifugation $(100,000 \mathrm{~g}$ for $2 \mathrm{hr})$, the clear supernatant was applied onto a $1-\mathrm{ml}$ column of ssDNA-agarose (BRL). After washing with $5 \mathrm{ml}$ of buffer $\mathrm{A}$, bound proteins were eluted with a $10-\mathrm{ml}$ $\mathrm{NaCl}$ gradient from 50 to $750 \mathrm{mM}$, and $0.5-\mathrm{ml}$ fractions were collected. For SDS-PAGE and immunoblotting, $10 \mu \mathrm{l}$ of the gradient fractions was analyzed in $9 \%$ gels for RAD18 and in $12 \%$ gels for RAD6.

\section{Acknowledgments}

V.B. and J.L. contributed equally to this work, which was supported by grants from the National Institutes of Health
(GM19261) and from the Department of Energy (DE-FG0393ER61706).

The publication costs of this article were defrayed in part by payment of page charges. This article must therefore be hereby marked "advertisement" in accordance with 18 USC section 1734 solely to indicate this fact.

\section{References}

Bailly, V., C.H. Sommers, P. Sung, L. Prakash, and S. Prakash. 1992. Specific complex formation between proteins encoded by the yeast DNA repair and recombination genes $R A D 1$ and RAD10. Proc. Nat. Acad. Sci. 89: 8273-8277.

Bowen, B., J. Steinberg, U.K. Laemmli, and H. Weintraub. 1980. The detection of DNA-binding proteins by protein blotting. Nucleic Acids Res. 8: 1-20.

Brill, S.J. and B. Stillman. 1989. Yeast replication factor-A functions in the unwinding of the SV40 origin of DNA replication. Nature 342: 92-95.

Dohmen, R.J., K. Madura, B. Bartel, and A. Varshavsky. 1991. The $\mathrm{N}$-end rule is mediated by the UBC2(RAD6) ubiquitinconjugating enzyme. Proc. Nat1. Acad. Sci. 88: 7351-7355.

Field, J., J.-I. Nikawa, D. Broek, B. MacDonald, L. Rodgers, I.A. Wilson, R.A. Lerner, and M. Wigler. 1988. Purification of a $R A S$-responsive adenyl cyclase complex from Saccharomyces cerevisiae by use of an epitope addition method. Mol. Cell. Biol. 8: 2159-2165.

Harlow, E. and D. Lane. eds. 1988. Antibodies: A laboratory manual. Cold Spring Harbor Laboratory, Cold Spring Harbor, NY.

Hershko, A. 1991. The ubiquitin pathway for protein degradation. Trends Biochem. Sci. 16: 265-268.

Jentsch, S. 1992. The ubiquitin-conjugation system. Annu. Rev. Genet. 26: 179-207.

Johnson, R.E., S.T. Henderson, T.D. Petes, S. Prakash, M. Bankmann, and L. Prakash. 1992. Saccharomyces cerevisiae $R A D 5$-encoded DNA repair protein contains DNA helicase and zinc-binding sequence motifs and affects the stability of simple repetitive sequences in the genome. Mol. Cell. Biol. 12: $3807-3818$.

Jones, J.S., S. Weber, and L. Prakash. 1988. The Saccharomyces cerevisiae RAD18 gene encodes a protein that contains potential zinc finger domains for nucleic acid binding and a putative nucleotide binding sequence. Nucleic Acids Res. 16: 7119-7131.

Koken, M.H., P. Reynolds, I. Jaspers-Dekker, L. Prakash, S. Prakash, D. Bootsma, and J.H. Hoeijmakers. 1991. Structural and functional conservation of two human homologs of the yeast DNA repair gene RAD6. Proc. Natl. Acad. Sci. 88: 8865-8869.

Laemmli, U.K. 1970. Cleavage of structural proteins during the assembly of the head of bacteriophage T4. Nature 227: 680685.

Lawrence, C.W. 1982. Mutagenesis in Saccharomyces cerevisiae. Adv. Genet. 21: 173-254.

Madura, K., R.J. Dohmen, and S. Varshavsky. 1993. N-recognin/ Ubc2 interactions in the $\mathrm{N}$-end rule pathway. J. Biol. Chem. 268: 12046-12054.

Prakash, L. 1981. Characterization of postreplication repair in Saccharomyces cerevisiae and effects of rad6, rad18, rev3 and rad52 mutations. Mol. Gen. Genet. 184: 471-478.

Prakash, S., P. Sung, and L. Prakash. 1993. DNA repair genes and proteins of Saccharomyces cerevisiae. Annu. Rev. Genet. 27: 33-70.

Reynolds, P., M.H. Koken, J.H. Hoeijmakers, S. Prakash, and L. 
Bailly et al.

Prakash. 1990. The rhp6 $6^{+}$gene of Schizosaccharomyces pombe: A structural and functional homolog of the RAD6 gene from the distantly related yeast Saccharomyces cerevisiae. EMBO I. 9: 1423-1430.

Sung, P., S. Prakash, and L. Prakash. 1988. The RAD6 protein of Saccharomyces cerevisiae polyubiquitinates histones, and its acidic domain mediates this activity. Genes \& Dev. 2: $1476-1485$

. 1990. Mutation of cysteine- 88 in the Saccharomyces cerevisiae RAD6 protein abolishes its ubiquitin-conjugating activity and its various biological functions. Proc. Natl. Acad. Sci. 87: 2695-2699.

Sung, P., E. Berleth, C. Pickart, S. Prakash, and L. Prakash 1991a. Yeast RAD6 encoded ubiquitin conjugating enzyme mediates protein degradation dependent on the $\mathrm{N}$-end-recognizing E3 enzyme. EMBO I. 10: 2187-2193.

Sung, P., S. Prakash, and L. Prakash. 1991b. Stable ester conjugate between the Saccharomyces cerevisiae RAD6 protein and ubiquitin has no biological activity. J. Mol. Biol. 221: 745-749.

Towbin, H., T. Staehelin, and J. Gordon. 1979. Electrophoretic transfer of proteins from polyacrylamide gels to nitrocellulose sheets: Procedure and some applications. Proc. Natl. Acad. Sci. 76: 4350-4354.

Watkins, J.F., P. Sung, S. Prakash, and L. Prakash. 1993. The extremely conserved amino terminus of RAD6 ubiquitinconjugating enzyme is essential for amino-end rule-dependent protein degradation. Genes \& Dev. 7: 250-261. 


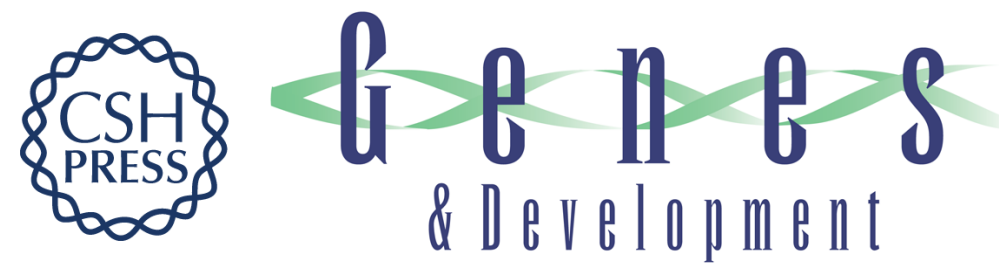

\section{Specific complex formation between yeast RAD6 and RAD18 proteins: a potential mechanism for targeting RAD6 ubiquitin-conjugating activity to DNA damage sites.}

V Bailly, J Lamb, P Sung, et al.

Genes Dev. 1994, 8:

Access the most recent version at doi:10.1101/gad.8.7.811

References This article cites 22 articles, 10 of which can be accessed free at: http://genesdev.cshlp.org/content/8/7/811.full.html\#ref-list-1

License

Email Alerting Service

Receive free email alerts when new articles cite this article - sign up in the box at the top right corner of the article or click here.

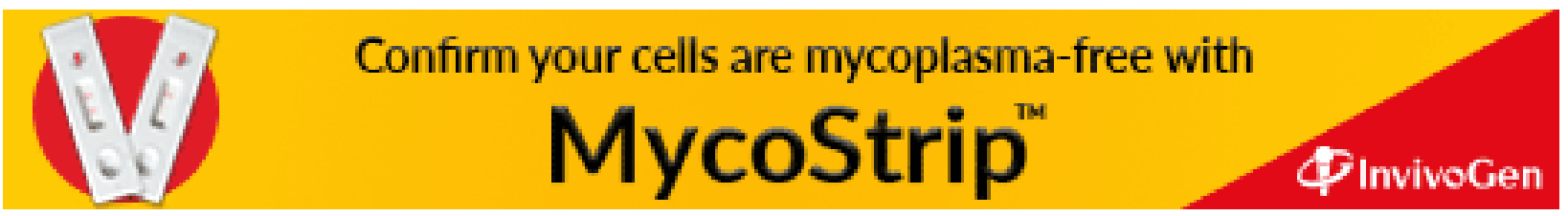

